\title{
The combination of NVP-BKM120 with trastuzumab or RAD001 synergistically inhibits the growth of breast cancer stem cells in vivo
}

\author{
FENG YU $^{1 *}$, JING ZHAO $^{1,2 *}$, YUNHUI HU $^{1}$, YANG ZHOU $^{1}$, RONG GUO $^{1}$, JINGCHAO BAI $^{1}$, \\ SHENG ZHANG ${ }^{1}$, HUILAI ZHANG ${ }^{2}$ and JIN ZHANG $^{1}$
}

\begin{abstract}
${ }^{1}$ The Third Department of Breast Cancer, China Tianjin Breast Cancer Prevention, Treatment and Research Center, Tianjin Medical University Cancer Institute and Hospital, National Clinical Research Center of Cancer, Tianjin 300060;

${ }^{2}$ Department of Lymphoma, Tianjin Medical University Cancer Hospital, Sino-US Center for Lymphoma and Leukemia, Tianjin Key Laboratory of Cancer Prevention and Therapy, Tianjin 300060, P.R. China
\end{abstract}

Received December 24, 2015; Accepted January 28, 2016

DOI: $10.3892 /$ or.2016.4799

\begin{abstract}
Deregulation of the phosphatidylinositol-3-kinase (PI3K)/Akt signalling pathway is common in breast cancer and is frequently associated with resistance to both traditional chemotherapy and targeted drugs. There is a growing body of evidence indicating that a small subpopulation of self-renewing cells, the so called cancer stem cells (CSC), are responsible for the growth of drug resistant secondary tumors. As many CSCs have upregulated the PI3K/Akt signalling pathway, preclinical and clinical studies are addressing the inhibition of this axis to target drug resistance. We evaluated the susceptibility of breast CSCs to NVP-BKM120 (BKM120), a new generation of PI3K-specific inhibitor, when used individually or in combination with trastuzumab or RAD001 both in vitro and in vivo. For this, a stem-like cell population (SC) was enriched from breast cancer cell lines after mammosphere cultures. We
\end{abstract}

Correspondence to: Professor Jin Zhang, The Third Department of Breast Cancer, Tianjin Medical University Cancer Institute and Hospital, Huan-Hu-Xi Road, Ti-Yuan-Bei, Hexi, Tianjin 300060, P.R. China

E-mail: zhangjin@tjmuch.com

*Contributed equally

Abbreviations: MTT, 3-(4,5-dimethylthiazol-2-yl)-2,5-diphenyltetrazolium bromide; ALDH, aldehyde dehydrogenase; bFGF, basic fibroblast growth factor; $\mathrm{CI}$, combination index; $\mathrm{IC}_{50}$; drug concentration necessary to kill $50 \%$ of cells; DMEM, Dulbecco's modified Eagle's medium; EGF, epidermal growth factor; mTOR, mammalian target of rapamycin; BKM120, NVP-BKM120; PBS, phosphate-buffered saline; RIPA, radioimmunoprecipitation assay; $\mathrm{SC}$, stem-like cell; TNBC, triple-negative breast cancer

Key words: NVP-BKM120, trastuzumab, RAD001, breast cancer stem cell, $\mathrm{PI} 3 \mathrm{~K} / \mathrm{Akt} / \mathrm{mTOR}$ demonstrated that BKM120 inhibits growth, generation of drug-resistant derivatives and $\mathrm{SC}$ formation in a panel of four breast cancer cell lines: MCF-7, MDA-MB-231, SK-BR-3 and CAL51. Importantly, BKM120 inhibits the PI3K/Akt signalling pathway in SCs from these cell lines. When BKM120 was used in combination with trastuzumab, a targeted therapy to treat HER2-positive breast cancer, we found synergistic cell growth inhibition, generation of drug resistant cells as well as SC formation from SK-BR-3 cells. Importantly, SK-BR-3 xenograft-derived tumors showed marginal growth when the drug combination was used. We also found a similar synergistic anticancer effect of BKM120 in combination with RAD001, an mTOR inhibitor, when treating triple-negative breast cancer cells in vitro and in both MDA-MB-231 and CAL51- mouse xenografts. Moreover, mouse data indicate that these drug combinations are well tolerated and provide the proof-of-concept and rationale to initiate clinical trials in both HER2-positive and triple-negative breast cancer.

\section{Introduction}

Breast cancer is the most frequent tumor in women and is the second cause of death from malignant diseases among women worldwide (1). Early diagnosis and development of targeted therapies have contributed to a reduction in breast cancer mortality. However, after an initial response to treatment, a high proportion of breast cancer patients become nonresponsive to therapy. This drug resistance phenomenon has been observed with both traditional and targeted therapies and is the main cause of breast cancer mortality $(2,3)$. Therefore, an understanding of the mechanisms of drug resistance is crucial for patient stratification and to develop novel targeted therapies. Cancer stem cells (CSCs), a small subpopulation of cancer cells with self-renewal, differentiation, and tumorigenic capabilities (4), have been suggested to explain many of the features of drug resistant tumors (5). CSCs have an increased resistance to a variety of chemotherapeutics in comparison to non-CSCs and are thought to drive tumor growth after an initial response to therapy (5). Although targeting CSCs is 
currently difficult, CSCs represent a promising target for novel anticancer drug development (6).

The phosphatidylinositol-3-kinase (PI3K) pathway plays a key role in the regulation of cell survival, growth, migration and proliferation of normal cells. Importantly, it is the most frequently misregulated signalling pathway in cancer (7). It has been implicated in the development, progression, and therapy resistance of breast cancer (8), mainly due to the activation of its major downstream effector Akt (9). PI3K $\alpha$ is a heterodimer with adaptor function, made up of a regulatory subunit of $85 \mathrm{kDa}$ $(\mathrm{p} 85 \alpha)$ and one of three possible catalytic subunits of $110 \mathrm{kDa}$ (p110) encoded by the PIK3CA (p110 $\alpha), P I K 3 C B(\mathrm{p} 110 \beta)$ and PIK3CD (p110 $\gamma$ ) genes (10). Activation of PI3K stimulates phosphorylation of phosphatidylinositol-4,5-diphosphate (PIP2), a phospholipid component of the cell membrane, and generation of phosphatidylinositol-3,4,5-triphosphate (PIP3), which bind pleckstrin homology domains of various signalling proteins. An inhibitory effect is exerted by the tumor suppressor PTEN (phosphatase and tensin homologue) which hydrolyzes and thus inactivates PIP3 (10). Importantly, activating mutations in the catalytic subunit of PI3K (p110 $\alpha$ ) and inactivating mutations in PTEN are frequently found in cancer (7).

NVP-BKM120 (BKM120) is a 2,6-dimorpholino pyrimidine derivative that is a potent pan-class I PI3K inhibitor, highly selective against other kinases including mammalian target of rapamycin (mTOR) (11). It shows anti-proliferative activity and induces apoptosis in cancer cell lines through inhibiting the PI3K/Akt signalling pathway $(12,13)$. Phase I clinical trials indicate that BKM120 is safe at the maximum-tolerated dose with a favourable pharmacokinetic profile in several solid tumors (14) and has been reported to overcome trastuzumab resistance in several breast cancer cell lines (15). Importantly, BKM120 has shown enhanced antitumor effect in mouse models when combined with inhibitors of other signalling pathways $(16,17)$.

An important downstream effector of PI3K/Akt is mTOR, a key activator of protein synthesis, a process which is frequently enhanced in cancer cells (18). Thus, the rapamycin analogue, and mTOR inhibitor, RAD001 (Everolimus) has gained attention as an anticancer agent and has been used in advanced renal cancer after failure of therapy to target vascular endothelial growth factor (19). Importantly, in addition to mTOR signalling inhibition, rapamycin analogues cause Akt activation and attenuation of their therapeutic efficacy $(20,21)$. Thus, it has been suggested that the combination of BKM120 and RAD001 may overcome these effects and has shown positive results in lung cancer mouse models (16).

In this study we demonstrate the efficacy of BKM120 combined with either trastuzumab or RAD001 targeting breast cancer stem cells. BKM120 displays antitumor activity by inhibiting the PI3K/Akt signalling pathway. The combination of BKM120 with either trastuzumab or RAD001 leads also to a decrease in the generation of drug resistant derivatives in vitro and excellent tumor response in xenograft mouse models.

\section{Materials and methods}

Cell lines and chemicals. Luminal A group MCF-7, claudin-low group triple-negative MDA-MB-231, triple-nega- tive CAL51 (22), and HER2 group trastuzumab-responsive SK-BR-3 breast cancer cell lines were used (23). Cells were maintained in Dulbecco's modified Eagle's medium (DMEM) supplemented with $1 \mathrm{~g} / \mathrm{l}$ glucose, $10 \%$ foetal calf serum and 4 mM L-glutamine (Life Technologies). NVB-BKM120 and RAD001 were a kind gift of Novartis. The HER2-inhibitor trastuzumab was obtained from Roche.

Flow cytometry. For stem cell markers, FITC-conjugated anti-CD44 and phycoerythrin-conjugated anti-CD24 antibodies, or their respective isotype controls, all from BD Biosciences were used essentially as described (24). An Aldefluor assay kit (StemCell Technologies) was used for the determination of aldehyde dehydrogenase (ALDH) activity by flow cytometry essentially as described (24). Briefly, cells were resuspended in assay buffer $\left(10^{6} \mathrm{cells} / \mathrm{ml}\right)$ and activated aldefluor substrate $(5 \mu \mathrm{l})$ was added to samples and incubated at $37^{\circ} \mathrm{C}$ for $45 \mathrm{~min}$ to allow substrate conversion. A sample with the ALDH inhibitor diethylaminobenzaldehyde was used as a negative control.

Mammosphere formation. Mammospheres were grown as described (24). In brief, cells $\left(1 \times 10^{3}\right)$ were plated in each well of an ultralow attachment 6-well plate (Corning) with $3 \mathrm{ml}$ serum-free mammary epithelial growth medium (MEGM; BioWhittaker), supplemented with 2\% B27 (Invitrogen), $20 \mathrm{ng} /$ $\mathrm{ml}$ EGF and $20 \mathrm{ng} / \mathrm{ml} \mathrm{bFGF}$ (BD Biosciences). Mammospheres were grown for 10 days and phase contrast images were obtained using Nikon TS100 microscope (Nikon, Shanghai, China). Where indicated, mammospheres were collected by centrifugation at $115 \mathrm{x}$ g for $10 \mathrm{~min}$ at room temperature, trypsinized, counted and used in further experiments (25).

Cell viability analysis. MTT [3-(4,5-dimethylthiazol-2-yl)2,5-diphenyltetrazolium bromide] assays were performed to evaluate the cell growth inhibitory effect in response to drug treatments and were used to determine the concentration of drug that inhibited cell growth by $50 \%\left(\mathrm{IC}_{50}\right)$ after 3 days of treatment (26). For drug combination experiments, a combination index (CI) was calculated using the CalcuSyn software (Biosoft) based on the Chou and Talalay method (27). CI values between 0.1 and 0.9 define different grades of synergism, with values between 0.9 and 1.1 being additive, whereas values $>1.1$ are antagonistic.

Drug resistance clonogenic assay. Cells (2x10 $/$ well of a 6-well plate) were treated with a single drug or a combination of drugs as indicated for 1 week. Drug resistant proliferating clones were fixed with $4 \%$ paraformaldehyde and stained with $0.2 \%$ crystal violet. Crystal violet retained within the cells was quantified by solubilization with $0.5 \%$ acetic acid and measurement of optical density at $592 \mathrm{~nm}$ (28).

Protein extraction and western blotting. A modified RIPA buffer (50 mM Tris-HCl, $150 \mathrm{mM} \mathrm{NaCl}, 0.25 \%$ SDS, $1 \%$ Triton $\mathrm{X}-100,0.25 \%$ sodium deoxycholate, $1 \mathrm{mM}$ EDTA, $1 \mathrm{mM}$ EGTA, $1 \mathrm{mM}$ dithiothreitol) with protease inhibitor cocktail (Sigma) was used for protein isolation from cells. Protein concentrations were determined using the BCA Protein Assay kit (Pierce). Cell lysates containing $50 \mu \mathrm{g}$ of protein were 

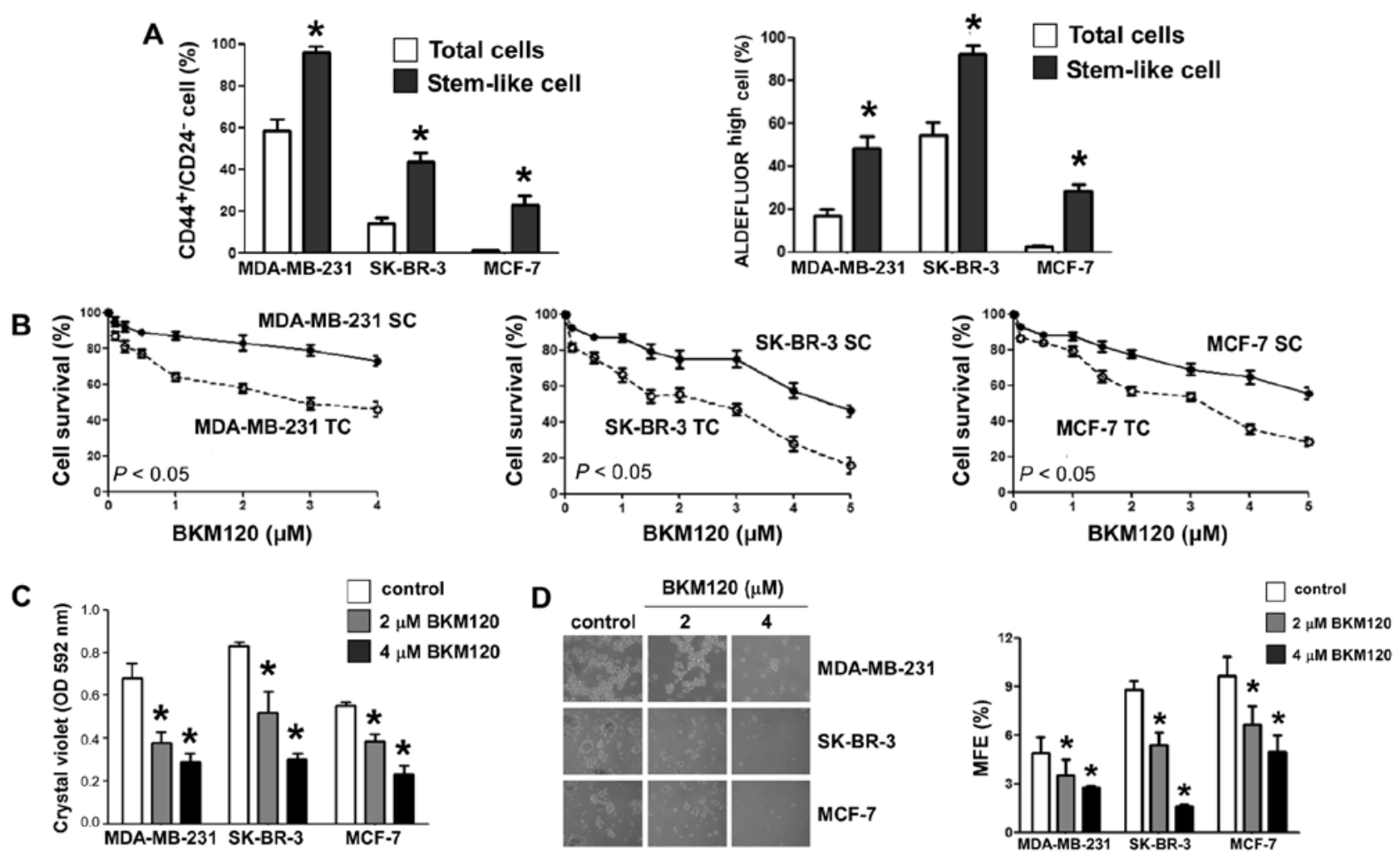

Figure 1. PI3K inhibitor BKM120 effectively inhibits the growth of breast cancer stem-like cells. (A) Quantitative flow cytometry data indicating the average percentages of $\mathrm{CD}_{4} 4^{+} / \mathrm{CD} 24^{-}$(left panel) and $\mathrm{ALDH}^{+}$cells (right panel) in breast cancer total cells and stem-like cells after their isolation from mammosphere cultures. (B) Dose-response curves of BKM120 for MDA-MA-231 (left panel), SK-BR-3 (middle panel) and MCF-7 (right panel) cells. In both cases, the total cell population (TC) and stem-like population (SC) were used. (C) Long-term drug resistance. Cells were treated with two doses of BKM120 for seven days and the cells were stained with crystal violet. Dye was solubilized and the optical density at $592 \mathrm{~nm}$ measured. (D) Mammosphere formation. Growth of mammospheres from MCF-7, SK-BR-3 and MDA-MB-231 cells in ultra low attachment plates in the presence / absence of BKM120. Mammosphere formation was visualized after 10 days at $\times 20$ magnification (left panel). Mammosphere forming efficacy (MFE) was calculated as the number of spheres formed in 10 days divided by the original number of single cells seeded and expressed as a percentage (right panel). Numerical data represent the mean \pm SD of at least three independent experiments $\left({ }^{*} \mathrm{P}<0.05\right)$. Pictorial data were repeated at least in triplicate and a representative picture is shown.

resolved on $12 \%(\mathrm{w} / \mathrm{v})$ polyacrylamide gels, transferred to nitrocellulose membranes (Millipore) and blocked with 5\% blotting grade milk (Bio-Rad) in PBST (0.1\% Tween-20 in PBS). Membranes were then incubated with primary antibodies to phospho-Akt (D9E), Akt1 (C73H10), phospho-S6 (D57.2.2E), S6 (54D2) and $\beta$-actin (13E5) (Cell Signaling Technology) at 1:1,000 dilution at $4^{\circ} \mathrm{C}$ overnight, followed by HRP-conjugated secondary antibodies (Cell Signaling Technology) at 1:2,000 dilution for $2 \mathrm{~h}$ at room temperature. Signals were visualized using SuperSignal West Pico Chemiluminescent substrate (Pierce) according to the manufacturer's instructions.

In vivo assays. Cells $\left(5 \times 10^{6}\right)$ were trypsinized and resuspended in a total volume of $100 \mu 1$ PBS containing 50\% Matrigel (BD Biosciences) and were injected into the mammary fat pad of nude mice (5-6 weeks of age). Tumor sizes were measured every three days in two dimensions using callipers, and the tumor volume calculated [tumor volume $\left(\mathrm{mm}^{3}\right)=$ $0.5 \mathrm{x} \mathrm{ab}^{2}$; $\mathrm{a}$ and $\mathrm{b}$ being the longest and shortest diameters of the tumor, respectively]. Fifteen days after cell injection, the tumor-bearing mice were randomly divided into four groups (five animals per group) and received: group 1, saline (control group); group 2, $50 \mathrm{mg} / \mathrm{kg}$ BKM120 (BKM120 group); group 3 , either $5 \mathrm{mg} / \mathrm{kg}$ trastuzumab (trastuzumab group) or $2 \mathrm{mg} / \mathrm{kg}$ RAD001 (RAD001 group); and group 4, a combination of BKM120 and either trastuzumab or RAD001 (at the above doses; combination group). Drugs were injected intraperitoneally every there days and tumor volume and mouse weight monitored until mice were sacrificed in a humane manner. All mice were maintained as required under the National Institutes of Health guidelines for the Care and Use of Laboratory Animals. The use of animals in this study was approved by the Animal Care and Use Committee of Tianjin Cancer Hospital.

Statistical analysis. Statistical evaluations were performed by Student's t-test for paired data and by ANOVA for sets of data with multiple comparison points. Statistical significance was considered at $\mathrm{p}<0.05$.

\section{Results}

BKM120 effectively inhibits the growth of breast cancer stem-like cells. Given the importance of the PI3K pathway in cancer, we asked whether BKM120 had a differential effect in the stem-like sub-populations from several breast cancer cell lines. Breast stem-like cells (SCs) exhibit the ability to survive and grow as mammospheres in low attachment plates $(29,30)$, are characterized by a CD $44^{+} / \mathrm{CD} 24 / \mathrm{ALDH} 1^{+}$ phenotype and show strong tumorigenicity in NOD/SCID 
Table I. Sensitivity of breast cancer cell lines to BKM120.

\begin{tabular}{lcrc}
\hline & \multicolumn{2}{c}{$\mathrm{IC}_{50}(\mu \mathrm{M})$} & \\
\cline { 2 - 3 } Cell line & \multicolumn{1}{c}{$\begin{array}{l}\text { Total } \\
\text { cells }\end{array}$} & $\begin{array}{c}\text { Stem-like } \\
\text { cells }\end{array}$ & $\begin{array}{l}\text { Stem-like cells } \\
\text { resistance ratio }\end{array}$ \\
\hline MCF-7 & $1.71 \pm 0.05$ & $7.29 \pm 0.51$ & 4.26 \\
MDA-MB-231 & $3.07 \pm 0.14$ & $20.01 \pm 3.46$ & 6.52 \\
SK-BR-3 & $1.64 \pm 0.16$ & $9.83 \pm 1.05$ & 5.99 \\
CAL51 & $1.21 \pm 0.12$ & $8.22 \pm 0.43$ & 6.79 \\
\hline
\end{tabular}

mouse models $(31,32)$. We isolated SCs from SK-BR-3, MDA-MB-231, MCF-7 and CAL51 cells after proliferation in low attachment plates. In all cases, the proportion of CD $44^{+} / \mathrm{CD} 24^{-}$and $\mathrm{ALDH} 1^{+}$cells was higher in SCs than in the original cell population (Figs. 1A and 5A). Next we tested the effect of BKM120 on cell survival using MTT assays. As expected, the pan-PI3K inhibitor had a dose-dependent effect on cell proliferation, both in the SC subpopulation and, to a greater extent, in the total cells (Figs. 1B and 5C). SCs resistance ratios varied between 4.26 in MCF-7 and 6.79 in CAL51 cells (Table I). Comparison with the $\mathrm{IC}_{50}$ obtained previously by us on similar cell sub-populations (33) indicates that BKM120 is more effective targeting SCs than docetaxel, a taxane type drug affecting cell proliferation by disruption of microtubules. As drug resistant cells have been proposed to arise from the selection of a small population of cells with stem-like properties (5), we asked whether BKM120 could inhibit the formation of drug resistant clones. For this, SCs were left to grow as monolayers up to one week with or without BMK120 and the cell mass determined by crystal violet staining. The pan-PI3K inhibitor decreased the proliferation of resistant cells in a dose-dependent manner in MDA-MB-231, MCF-7 and SK-BR-3 cells (Fig. 1C). As BKM120 inhibited growth on monolayer cultures, we also performed mammosphere forming assays to detect whether BKM120 could eliminate SC growth. Indeed, the mammosphere-forming efficiency (MFE) decreased in a dose-dependent manner in MDA-MB-231, SK-BR-3, MCF-7 (Fig. 1D) and CAL51 (Fig. 5E) cells. Thus, BKM120 inhibits the growth of breast cancer SCs.

BKM120 effectively inhibits the PI3K/Akt/mTOR signalling pathway. As BKM120 is a pan-PI3K inhibitor, we next sought to determine its effect on the PI3K/Akt/mTOR axis in breast cancer cells. For this, we analyzed by western blotting the total and phosphorylated levels of Akt and ribosomal protein S6, both in SCs and the total cellular population. Both phosphorylated Akt and S6 levels were higher in SCs than in the whole cell population, although there were variations among cells. MCF-7 and SK-BR-3 SCs showed higher activation of Akt than MDA-MB-231 SCs. Ribosomal protein S6 was clearly activated in SK-BR-3 SCs, although the activation was less robust in the other two SC subpopulations (Fig. 2A).

Having confirmed an activation of the PI3K/Akt/mTOR pathway in SCs, we treated both SCs and the whole cell population with a range of BKM120 concentrations for $24 \mathrm{~h}$ and determined the extent of the above proteins by western blot-

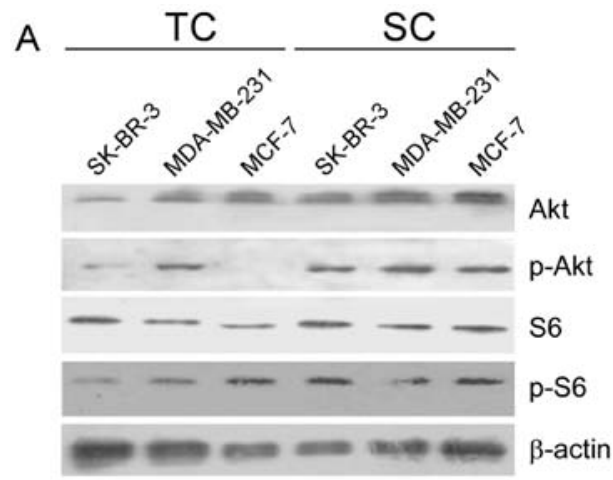

B
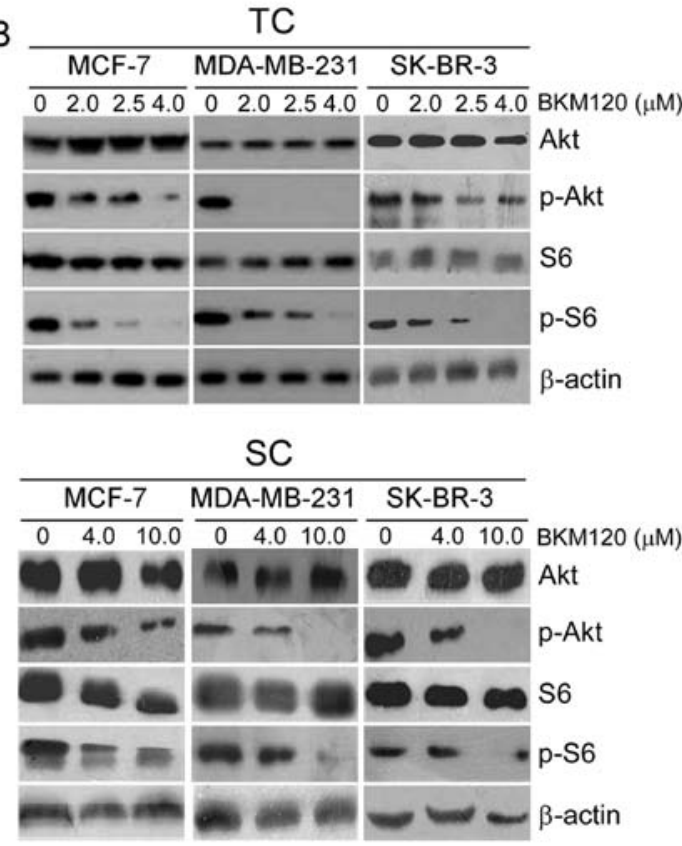

Figure 2. BKM120 effectively inhibits the PI3K/Akt pathway in breast cancer cell lines and stem-like cell subpopulations. (A) The PI3K/Akt signalling pathway is activated in SCs. Akt, ribosomal protein S6, and their phosphorylated forms were detected by western blotting in extracts of either total cells or their corresponding SC subpopulation. $\beta$-actin was used as a loading control. (B) Effect of BKM120 on the PI3K/Akt signaling pathway in MDA-MB-231 and SK-BR-3 cells. Cells were treated for $24 \mathrm{~h}$ with BKM120 prior to western blotting. Data show representative blots of at least three different experiments.

ting. As expected, higher doses of BKM120 were necessary to decrease the levels of phospho-Akt and phospho-S6 in SCs than in the whole cell population. For instance, $2 \mu \mathrm{M}$ BKM120 completely inhibited the Akt pathway in MDA-MB-231 cells whereas in the SC subpopulation a partial inhibition was obtained after treatment with $4 \mu \mathrm{M}$ and a total inhibition was achieved only with $10 \mu \mathrm{M}$ BKM120. Similarly, phospho-S6 was absent in cells treated with up to $4 \mu \mathrm{M}$ BKM120, whereas $10 \mu \mathrm{M}$ BKM120 partly inhibited S6 activation in SCs (Fig. 2B).

Therefore, BKM120 exerts potent suppressive effects on PI3K/Akt/mTOR signalling in both total and SCs subpopulations of breast cancer cells.

The combination of BKM120 and trastuzumab synergistically inhibits the growth of SK-BR-3 cells and eliminates the SC subpopulation. Alterations in the PI3K/Akt signalling pathway have been associated with therapy-induced resistance in 

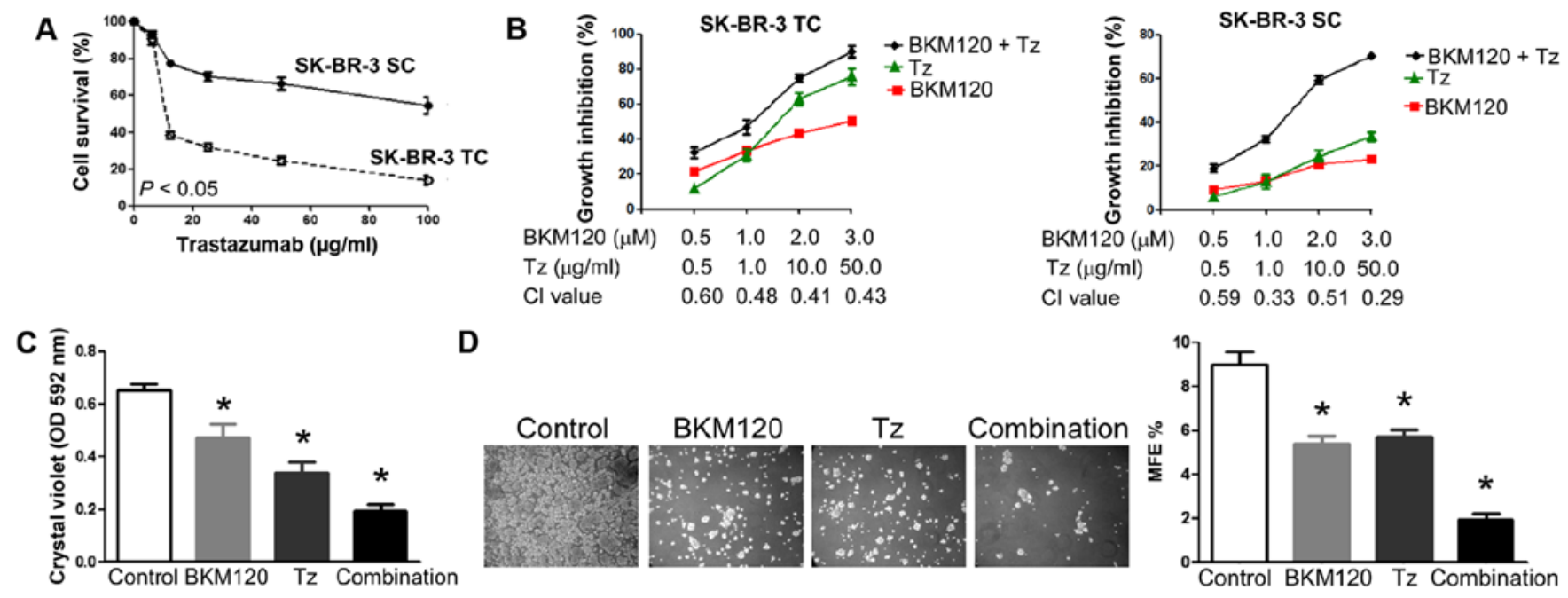

D
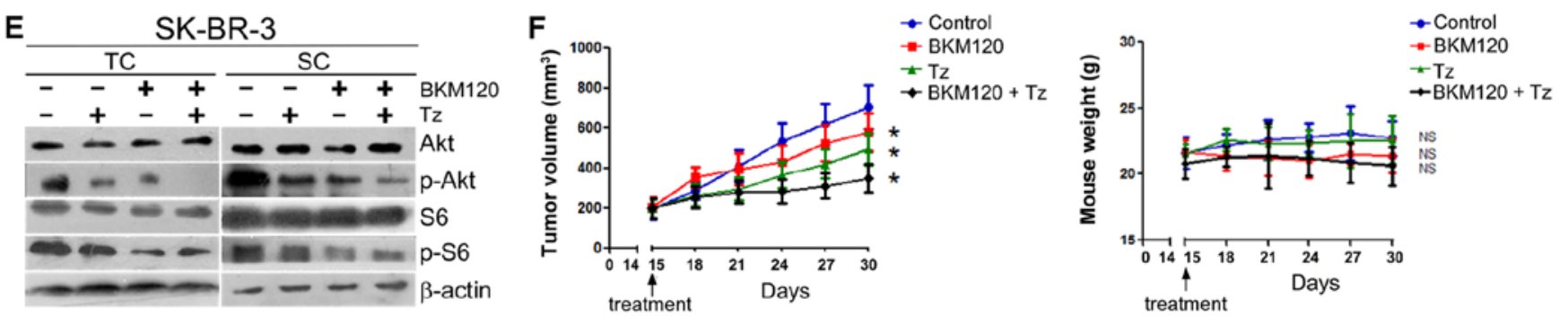

Figure 3. The combination of BKM120 and trastuzumab synergistically inhibits the growth of HER $2^{+} \mathrm{SCs}$ in vitro and the formation of tumors in vivo. (A) Dose-response curves of trastuzumab for SK-BR-3 cells. TC, total cell population; SC, stem-like population. (B) The combination of BKM120 and trastuzumab synergistically inhibits the growth of SK-BR-3 TCs (left panel) and SK-BR-3 SCs (right panel). Cells were treated with different combinations of BKM120 and trastuzumab for three days and the effect on cellular proliferation determined by MTT assays. Combination index $(C I)$ for each set of drug

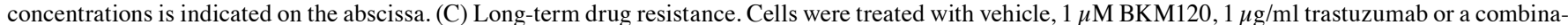
tion of the two drugs for seven days and stained with crystal violet. After dye solubilization, the optical density at $592 \mathrm{~nm}$ was determined. (D) Effect of a combination of trastuzumab and BKM120 on the growth of mammospheres from SK-BR-3 cells. Cells were treated with vehicle, $1 \mu \mathrm{M}$ BKM120, $1 \mu \mathrm{g} / \mathrm{ml}$ trastuzumab or a combination of the two drugs for ten days. Mammosphere formation was visualized at x 20 magnification (left panel) and MFE calculated (right panel). (E) Effect of BKM120 and trastuzumab on Akt and S6 phosphorylation in SK-BR-3 cells. Both TCs and SCs were treated with $100 \mu \mathrm{g} / \mathrm{ml}$ trastuzumab in the absence or presence of $4 \mu \mathrm{M}$ BKM120 for $24 \mathrm{~h}$ and the levels of Akt, S6 and their phosphorylated forms analyzed by western blotting. $\beta$-actin was used as a loading control. (F) Antitumor activity of BKM120 combined with trastuzumab in SK-BR-3 xenograft tumors (right panel). Growth of SK-BR-3 xenograft tumors (expressed as tumor volume) after treatment with PBS (control), trastuzumab, BKM120, or BKM120 plus trastuzumab (left panel, see Materials and methods for experimental details). Data represent mean tumor size \pm SD of five tumors per group. Right panel illustrates body weight of nude mice bearing SK-BR-3 xenografts. Data indicate mean body weight \pm SD of five mice per group. Other numerical data represent the mean \pm SD of three independent experiments (" $\mathrm{P}<0.05$; NS, not significant). Pictorial data were repeated at least in triplicate and a representative picture is shown.

breast cancer patients, including endocrine-based therapy and combined chemotherapy and HER2-targeted-therapy $(34,35)$. Recent studies demonstrate that targeting the PI3K/Akt pathway in combination with trastuzumab, a monoclonal antibody that interferes with the HER2/neu receptor, is beneficial in trastuzumab-resistant breast cancer (15). As BKM120 has been shown to have robust anticancer properties in breast cancer SCs, we asked whether BKM120 could synergize with trastuzumab in SK-BR-3, a HER2 ${ }^{+}$breast cancer cell line, especially in its SC subpopulation. For this, SK-BR-3 total cells and SCs were treated with increasing concentrations of trastuzumab, either alone or in combination with BKM120. As expected, trastuzumab decreased cell survival, although the effect was more noticeable in the whole SK-BR-3 population $\left(\mathrm{IC}_{50} \sim 10 \mu \mathrm{g} / \mathrm{ml}\right)$ than in SCs $\left(\mathrm{IC}_{50}>100 \mu \mathrm{g} / \mathrm{ml}\right.$ ) (Fig. 3A). When used in combination, the $\mathrm{CI}$ values ranged from 0.3 to 0.6 (Fig. 3B), indicating that trastuzumab and BKM120 act synergistically both in total SK-BR-3 cells and SCs (27). Importantly, trastuzumab in combination with BKM120 had a greater effect suppressing the generation of resistant cells (Fig. 3C) and mammospheres (SCs; Fig. 3D) than the individual drugs acting alone. Western blot analyses also indicated a stronger effect on the PI3K/Akt/mTOR pathway when the drugs were combined. Both phospho-Akt and phospho-S6 levels decreased to a high extent in SCs, whilst, as expected, the effect was stronger in the whole cell population (Fig. 3E).

Next, we used a xenograft model to assess the efficacy of this drug combination against the growth of SK-BR-3-derived tumors. For this, SK-BR-3 SCs were injected into the mammary fat pad of female nude mice. Mice were then randomly divided into four groups 14 days after injection and treated with vehicle, BKM120, trastuzumab, and a combination of the two. As expected, tumor growth followed a steady progress during the following 15 days in the control group, whereas the tumor volume increased at lower rates in the BKM120 and trastuzumab groups. Importantly, the group receiving both BKM120 and trastuzumab showed a slight tumor growth 

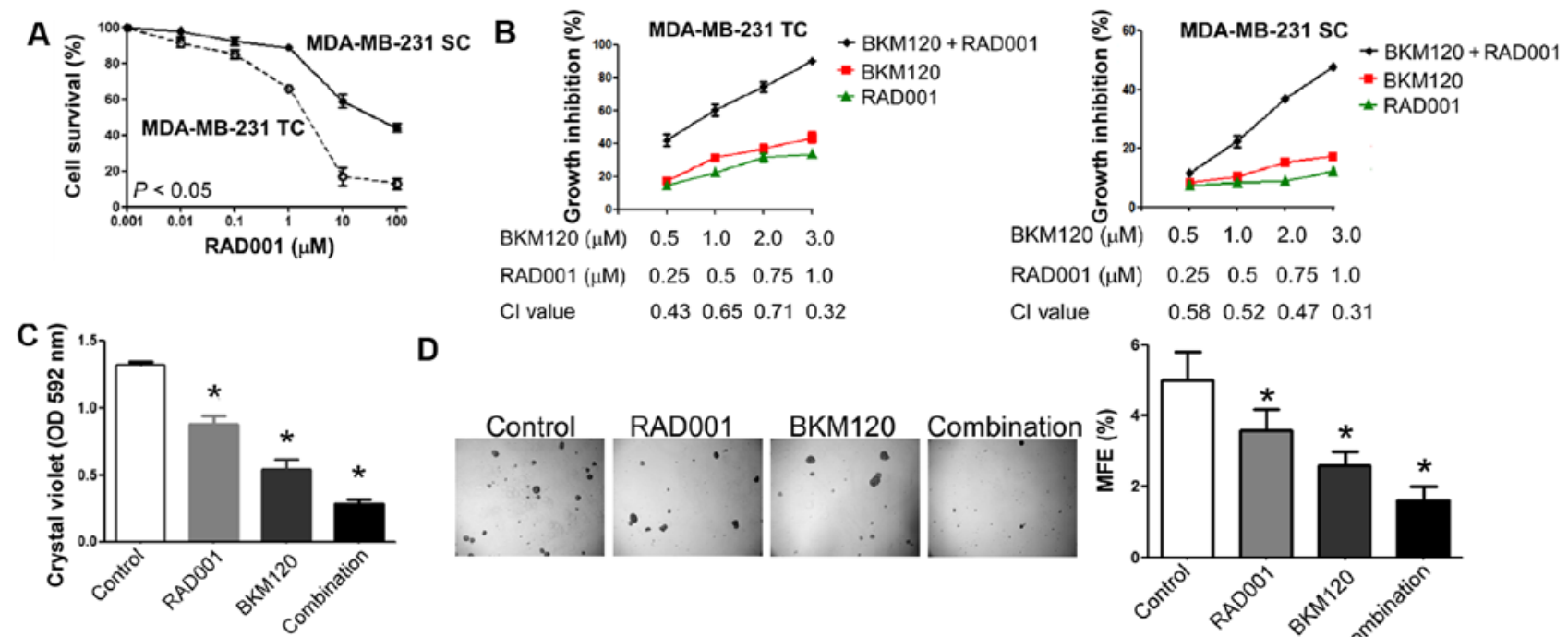

RAD001 $(\mu \mathrm{M}) \quad 0.25 \quad 0.5 \quad 0.75 \quad 1.0$

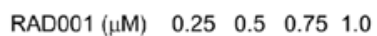

$\begin{array}{llllllll}C l & \text { value } \quad 0.43 & 0.65 & 0.71 & 0.32\end{array}$

$\mathrm{Cl}$ value

$\begin{array}{llll}0.58 & 0.52 & 0.47 & 0.31\end{array}$
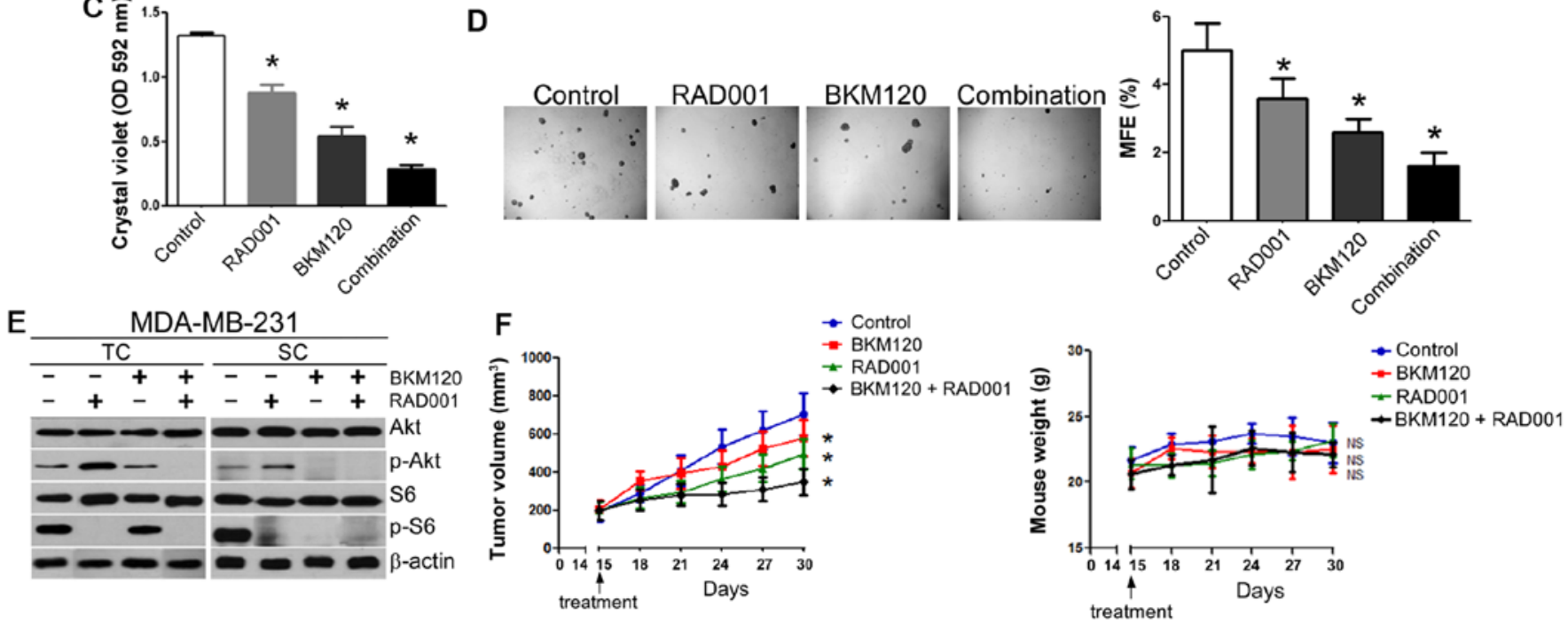

Figure 4. The combination of BKM120 and RAD001 synergistically inhibits the growth of triple negative MDA-MB-231 cells both in vitro and in vivo. (A) Dose-response curves of RAD001 for MDA-MB-231 cells. TC, total cell population; SC, stem-like population. (B) The combination of BKM120 and RAD001 synergistically inhibits the growth of MDA-MB-231 TCs (left panel) and MDA-MB-231 SCs (right panel). Cells were treated with different combinations of BKM120 and RAD001 for three days and the effect on cellular proliferation determined by MTT assays. Combination index (CI) for each set of drug concentrations is indicated on the abscissa. (C) Long-term drug resistance. Cells were treated with vehicle, $1 \mu \mathrm{M}$ BKM120, $0.5 \mu \mathrm{M}$ RAD001 or a combination of the two drugs for seven days and stained with crystal violet. After dye solubilization, the optical density at $592 \mathrm{~nm}$ was determined. (D) Effect of a combination of RAD001 and BKM120 on the growth of mammospheres from MDA-MB-231 cells. Cells were treated with vehicle, $1 \mu \mathrm{M}$ BKM120, $0.5 \mu \mathrm{M}$ RAD001 or a combination of the two drugs for ten days. Mammosphere formation was visualized at x20 magnification (left panel) and MFE calculated (right panel). (E) Effect of BKM120 and RAD001 on Akt and S6 phosphorylation in MDA-MB-231 cells. Both TCs (left panel) and SCs (right panel) were treated with $1 \mu$ M RAD001 in the absence or presence of $3 \mu \mathrm{M}$ BKM120 for $24 \mathrm{~h}$ and the levels of Akt, S6 and their phosphorylated forms analyzed by western blotting. $\beta$-actin was used as a loading control. (F) Antitumor activity of BKM120 combined with RAD001 in MDA-MB-231 xenograft tumors (right panel). Growth of MDA-MB-231 xenograft tumors (expressed as tumor volume) after treatment with PBS (control), RAD001, BKM120, or BKM120 plus RAD001 (left panel, see Materials and methods for experimental details). Data represent mean tumor size \pm SD of five tumors per group. Right panel illustrates body weight of nude mice bearing MDA-MB-231 xenografts. Data indicate mean body weight \pm SD of five mice per group. Other numerical data represent the mean \pm SD of three independent experiments ("P<0.05; NS, not significant). Pictorial data were repeated at least in triplicate and a representative picture is shown.

with no significant mouse body weight loss, indicating that the drug combination is well tolerated (Fig. 3F).

In summary, BKM120 in combination with trastuzumab acts synergistically inhibiting the PI3K/Akt/mTOR pathway, the growth of HER $2^{+}$cells, the generation of drug-resistant SCs in vitro, and the formation of tumors in vivo.

The combination of BKM120 and RAD001 synergistically inhibits the growth of triple-negative breast cancer cells both in vitro and in vivo. We have previously demonstrated that the combination of RAD001 with docetaxel, latrozole or trastuzumab has enhanced growth-inhibitory effects against breast cancer SCs $(33,36,37)$. As RAD001 has been reported to act synergistically with BKM120 in lung cancer models (16), we asked whether a similar effect would be observed in triple-negative breast cancer (TNBC). TNBC remains a challenging clinical problem due to a lack of targeted therapies and, consequently, a high mortality rate. For this purpose, total cells and SCs of MDA-MB-213 and CAL51 cell lines were treated with increasing concentrations of RAD001, either alone or in combination with a range of doses of BKM120. As expected, RAD001 decreased cell survival, although the effect was more noticeable in the total cell population than in SCs (Figs. 4A and 5B). When used in combination, RAD001 and BKM120 CI values ranged from 0.3 to 0.7 in MDA-MB-231 (Fig. 4B) and from 0.2 to 0.8 and CAL51 cells (Fig. 5C). This indicates that RAD001 and BKM120 act synergistically when used in combination, both in the total population and in the SC subpopulations of these two cell lines (27). Importantly, RAD001 in combination with BKM120 had a greater effect on the generation of resistant cells (Figs. 4C and 5D) and mammosphere formation (Figs. 4D and 5E) than the drugs individually.

Inhibition of mTOR leads to feedback reactivation of PI3K activity in a variety of systems $(21,38)$. We confirmed that RAD001 treatment, as a single agent, increased Akt phoshphorylation in MDA-MB-231 (Fig. 4E) and CAL51 cells (Fig. 5F). However, RAD001 failed to activate PI3K activity in the presence of BKM120, both in the total cell popu- 

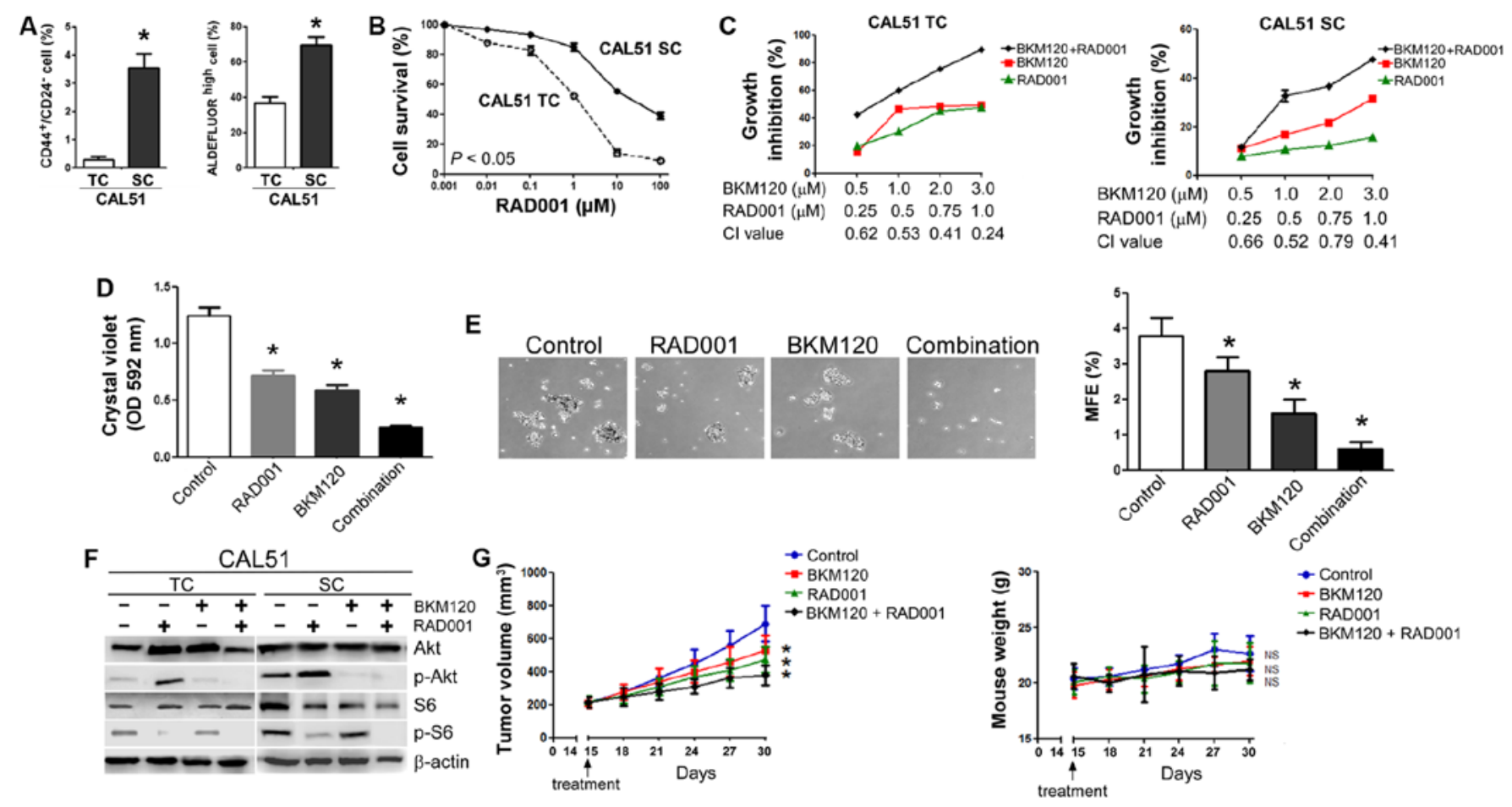

Figure 5. The combination of BKM120 and RAD001 synergistically inhibits the growth of triple-negative CAL51 cells both in vitro and in vivo. (A) Quantitative flow cytometry data indicating the average percentages of $\mathrm{CD} 44^{+} / \mathrm{CD} 24^{-}$(left panel) and ALDH ${ }^{+}$cells (right panel) in CAL51 total cells (TC) and stem-like cells $(S C)$ after their isolation by mammosphere cultures. (B) Dose-response curves of RAD001. (C) The combination of BKM120 and RAD001 synergistically inhibits the growth of CAL51 TCs (left panel) and CAL51 SCs (right panel). Cells were treated with different combinations of BKM120 and RAD001 for three days and the effect on cellular proliferation determined by MTT assays. Combination index $(\mathrm{CI})$ for each set of drug concentrations is indicated on the abscissa. (D) Long-term drug resistance. Cells were treated with vehicle, $1 \mu \mathrm{M}$ BKM120, $0.5 \mu \mathrm{M}$ RAD001 or a combination of the two drugs for seven days and stained with crystal violet. After dye solubilization, the optical density at $592 \mathrm{~nm}$ was determined. (E) Effect of a combination of RAD001 and BKM120 on the growth of mammospheres from CAL51 cells. Cells were treated with vehicle, $1 \mu \mathrm{M}$ BKM120, $0.5 \mu \mathrm{M}$ RAD001 or a combination of the two drugs for ten days. Mammosphere formation was visualized at x20 magnification (left panel) and MFE calculated (right panel). (F) Effect of BKM120 and RAD001 on Akt and S6 phosphorylation in CAL51 cells. Both TCs (left panel) and SCs (right panel) were treated with $1 \mu \mathrm{M}$ RAD001 in the absence or presence of $1 \mu \mathrm{M}$ BKM120 for $24 \mathrm{~h}$ and the levels of Akt, S6 and their phosphorylated forms analyzed by western blotting. $\beta$-actin was used as a loading control. (G) Antitumor activity of BKM120 combined with RAD001 in CAL51 xenograft tumors. Growth of CAL51 xenograft tumors (expressed as tumor volume) after treatment with PBS (control), RAD001, BKM120, or BKM120 plus RAD001 (left panel, see Materials and methods for experimental details). Data represent mean tumor size \pm SD of five tumors per group. Right panel illustrates body weight of nude mice bearing CAL51 xenografts. Data indicate mean body weight \pm SD of five mice per group. Other numerical data represent the mean $\pm \mathrm{SD}$ of three independent experiments (" $\mathrm{P}<0.05$; NS, not significant). Pictorial data were repeated at least in triplicate and a representative picture is shown.

lation and SCs. The combination of BKM120 and RAD001 also showed more activity in reducing phospho-S6 levels than either single agent did when acting alone (Figs. 4E and 5F). Thus, the combination of BKM120 and RAD001 blocks RAD001-induced phosphorylation of Akt and exerts enhanced effects on suppression of phospho-S6.

Because of the growth-inhibitory effects of the BKM120 and RAD001 combination in TNBC SCs in vitro (Figs. 4B and $5 \mathrm{C}$ ), we sought to determine whether the same effect could be found in vivo. For this, MDA-MB-231 SCs or CAL51 SCs were injected into the mammary fat pad of female nude mice. Mice were then randomly divided into four groups 14 days after injection and treated with vehicle, BKM120, RAD001, and a combination of the two drugs. As expected, tumor growth followed a steady progress during the following 15 days in the control group, whereas the tumor volume increased at lower rates in the BKM120 and RAD001 groups. Importantly, the group receiving both BKM120 and RAD001 showed the slowest tumor growth and absence of mouse body weight loss, indicating that the drug combination is well tolerated (Figs. $4 \mathrm{~F}$ and $5 \mathrm{G}$ ).
In summary, BKM120 in combination with RAD001 acts synergistically inhibiting the PI3K/Akt/mTOR pathway, the growth of TNBC cells, the generation of drug-resistant derivatives in vitro, and the formation of tumors in vivo.

\section{Discussion}

The development of cancer targeted therapies has been of paramount importance for the increase in patient survival achieved during the last few decades $(39,40)$. However, most targeted therapies, as well as traditional non-targeted cytotoxic and radiation therapies, are encumbered by the acquisition of resistance. Accumulating evidence indicates that CSCs play a crucial role in therapy resistance and recurrence of breast cancers $(5,32)$. Therapy resistance is a complex phenomenon involving multiple mechanisms, including activation of signalling pathways such as the PI3K/Akt/mTOR axis (41), and the activation of this pathway is crucial for maintaining the stemness and chemoresistance of breast CSCs (42). Hence, breast CSCs are critical therapeutic targets and their elimination may improve the prognosis and outcome of cancer therapy (43). PI3K inhibition has 
recently been shown to sensitize CSCs to chemotherapy and targeted therapy in several cancers including leukemia (44), hepatocellular carcinoma (45) and breast cancer (33). In line with these studies, we present data indicating that the PI3K inhibitor BKM120 is also effective in eliminating breast CSCs. After BKM120 treatment, the in vitro tumorigenicity of breast cancer cells is highly impaired. Moreover, BKM120 exerts tumor inhibiting effect on breast SCs-derived xenograft models in vivo, further confirming the potency of BKM120 in CSCs.

Aberrant activation of several signalling pathways downstream of HER2, including the MAPK (46), Notch (47) and PI3K/Akt pathways (48), leads to HER2-targeted therapy resistance. Since HER2 mediates signal transduction through the PI3K/Akt pathway, inhibition of components of this pathway is a reasonable approach to overcome resistance to HER2-targeted therapy $(15,49)$. Indeed, the combination of PI3K inhibitor BAY 80-6946 with HER2-targeted therapy inhibits HER2positive breast cancer cell growth more effectively than either therapy used alone (50), and the phase Ib study of BKM120 plus trastuzumab in HER2-positive breast cancer patients has shown promising results (51). Here, we report that BKM120 has a synergistic effect with trastuzumab on HER2-positive breast SCs in vitro and, importantly, that the drug combination is well tolerated in mouse models. This adds weight to the design of future trials with a combination of BKM120 and transtuzumab in HER2-positive breast cancer patients.

TNBC is a heterogeneous disease comprised of several biologically distinct subtypes (52). In addition to our poor understanding of the molecular characteristics of each TNBC subtype, we lack effective targeting strategies, leading to a poor prognosis for TNBC patients. However, anti-angiogenic, EGFRtargeted, PARP inhibitors, PI3K/Akt/mTOR inhibitors and Src inhibiting therapies have demonstrated promising results (53). It has been suggested that TNBC patients might benefit from the combined effect of BKM120 with PARP inhibitors, as BKM120 sensitizes BRCA-proficient TNBC to PARP inhibition (54). Experimental data indicate that RAD001 has favourable activity against basal-like TNBCs (55) and phase 2 clinical trials show that RAD001 combined with carboplatin is efficacious in metastatic TNBC (56). Here, we demonstrated that the combination of BKM120 and RAD001 enhances the suppressive growth effect of TNBC cells, including SCs both in vitro and in vivo. It is also important to emphasize that BKM120 inhibits RAD001induced Akt phosphorylation in TNBC, in line with studies using other systems $(21,57)$. Accordingly, we suggest that the combination of BKM120 and RAD001 may be an effective regimen for TNBC treatment.

In summary, we demonstrate that the combination of a panPI3K inhibitor, BKM120, with either trastuzumab or RAD001, is effective in targeting breast cancer SCs in vivo and offers the rationale to develop further clinical trials for HER2-positive and TNBC, respectively.

\section{Acknowledgements}

This study was supported by the Chinese National Natural Sciences Foundation (81402480 to Y.H.), Tianjin municipal Major Scientific and Technological Special Project for Significant Anticancer Development (12ZCDZSY15700 to J. Zhang), Tianjin municipal Natural Sciences Foundation
(15JCYBJC28300 to S.Z.) and Tianjin Medical University Cancer Institute and Hospital Foundation (1416 to J. Zhao).

\section{References}

1. Mendes D, Alves C, Afonso N, Cardoso F, Passos-Coelho JL, Costa L, Andrade S and Batel-Marques F: The benefit of HER2-targeted therapies on overall survival of patients with metastatic HER2positive breast cancer - a systematic review. Breast Cancer Res 17: $140,2015$.

2. Fan W, Chang $\mathrm{J}$ and $\mathrm{Fu} \mathrm{P}$ : Endocrine therapy resistance in breast cancer: Current status, possible mechanisms and overcoming strategies. Future Med Chem 7: 1511-1519, 2015

3. Chen Z, Shi T, Zhang L, Zhu P, Deng M, Huang C, Hu T, Jiang L and Li J: Mammalian drug efflux transporters of the ATP binding cassette $(\mathrm{ABC})$ family in multidrug resistance: A review of the past decade. Cancer Lett 370: 153-164, 2016.

4. Ailles LE and Weissman IL: Cancer stem cells in solid tumors. Curr Opin Biotechnol 18: 460-466, 2007.

5. Dean M, Fojo T and Bates S: Tumour stem cells and drug resistance. Nat Rev Cancer 5: 275-284, 2005.

6. Takebe N, Harris PJ, Warren RQ and Ivy SP: Targeting cancer stem cells by inhibiting Wnt, Notch, and Hedgehog pathways. Nat Rev Clin Oncol 8: 97-106, 2011.

7. Yuan TL and Cantley LC: PI3K pathway alterations in cancer: Variations on a theme. Oncogene 27: 5497-5510, 2008.

8. Baselga J: Targeting the phosphoinositide-3 (PI3) kinase pathway in breast cancer. Oncologist 16 (Suppl 1): 12-19, 2011.

9. Burris HA III: Overcoming acquired resistance to anticancer therapy: Focus on the PI3K/AKT/mTOR pathway. Cancer Chemother Pharmacol 71: 829-842, 2013.

10. Engelman JA, Luo J and Cantley LC: The evolution of phosphatidylinositol 3-kinases as regulators of growth and metabolism. Nat Rev Genet 7: 606-619, 2006.

11. Maira SM, Pecchi S, Huang A, Burger M, Knapp M, Sterker D, Schnell C, Guthy D, Nagel T, Wiesmann M, et al: Identification and characterization of NVP-BKM120, an orally available pan-class I PI3-kinase inhibitor. Mol Cancer Ther 11: 317-328, 2012.

12. Zheng Y, Yang J, Qian J, Zhang L, Lu Y, Li H, Lin H, Lan Y, Liu Z, He J, et al: Novel phosphatidylinositol 3-kinase inhibitor NVP-BKM120 induces apoptosis in myeloma cells and shows synergistic anti-myeloma activity with dexamethasone. J Mol Med Berl 90: 695-706, 2012.

13. Amrein L, Shawi M, Grenier J, Aloyz R and Panasci L: The phosphatidylinositol-3 kinase I inhibitor BKM120 induces cell death in B-chronic lymphocytic leukemia cells in vitro. Int J Cancer 133: 247-252, 2013.

14. Bendell JC, Rodon J, Burris HA, de Jonge M, Verweij J, Birle D, Demanse D, De Buck SS, Ru QC, Peters M, et al: Phase I, dose-escalation study of BKM120, an oral pan-Class I PI3K inhibitor, in patients with advanced solid tumors. J Clin Oncol 30: 282-290, 2012.

15. O'Brien NA, McDonald K, Tong L, von Euw E, Kalous O, Conklin D, Hurvitz SA, di Tomaso E, Schnell C, Linnartz R, et al: Targeting $\mathrm{PI} 3 \mathrm{~K} / \mathrm{mTOR}$ overcomes resistance to HER2-targeted therapy independent of feedback activation of AKT. Clin Cancer Res 20: 3507-3520, 2014.

16. Ren H, Chen M, Yue P, Tao H, Owonikoko TK, Ramalingam SS, Khuri FR and Sun SY: The combination of RAD001 and NVP-BKM120 synergistically inhibits the growth of lung cancer in vitro and in vivo. Cancer Lett 325: 139-146, 2012.

17. Jane EP, Premkumar DR, Morales A, Foster KA and Pollack IF: Inhibition of phosphatidylinositol 3-kinase/AKT signaling by NVP-BKM120 promotes ABT-737-induced toxicity in a caspasedependent manner through mitochondrial dysfunction and DNA damage response in established and primary cultured glioblastoma cells. J Pharmacol Exp Ther 350: 22-35, 2014.

18. Silvera D, Formenti SC and Schneider RJ: Translational control in cancer. Nat Rev Cancer 10: 254-266, 2010.

19. Albiges L, Kube U, Eymard JC, Schmidinger M, Bamias A, Kelkouli N, Mraz B, Florini S, Guderian G, Cattaneo A, et al: Everolimus for patients with metastatic renal cell carcinoma refractory to anti-VEGF therapy: Results of a pooled analysis of non-interventional studies. Eur J Cancer 51: 2368-2374, 2015.

20. O'Reilly KE, Rojo F, She QB, Solit D, Mills GB, Smith D, Lane H, Hofmann F, Hicklin DJ, Ludwig DL, et al: mTOR inhibition induces upstream receptor tyrosine kinase signaling and activates Akt. Cancer Res 66: 1500-1508, 2006. 
21. Wang X, Yue P, Kim YA, Fu H, Khuri FR and Sun SY: Enhancing mammalian target of rapamycin (mTOR)-targeted cancer therapy by preventing $\mathrm{mTOR} /$ raptor inhibition-initiated, mTOR/rictor-independent Akt activation. Cancer Res 68: 7409-7418, 2008.

22. Gioanni J, Le François D, Zanghellini E, Mazeau C, Ettore F, Lambert JC, Schneider M and Dutrillaux B: Establishment and characterisation of a new tumorigenic cell line with a normal karyotype derived from a human breast adenocarcinoma. Br J Cancer 62: 8-13, 1990.

23. Holliday DL and Speirs V: Choosing the right cell line for breast cancer research. Breast Cancer Res 13: 215, 2011.

24. Lombardo Y, Filipović A, Molyneux G, Periyasamy M, Giamas G, Hu Y, Trivedi PS, Wang J, Yagüe E, Michel L, et al: Nicastrin regulates breast cancer stem cell properties and tumor growth in vitro and in vivo. Proc Natl Acad Sci USA 109: 16558-16563, 2012.

25. Lombardo Y, de Giorgio A, Coombes CR, Stebbing J and Castellano L: Mammosphere formation assay from human breast cancer tissues and cell lines. J Vis Exp 97: e52671, 2015.

26. Hu Y, Cheng X, Li S, Zhou Y, Wang J, Cheng T, Yang M and Xiong D: Inhibition of sorcin reverses multidrug resistance of K562/A02 cells and MCF-7/A02 cells via regulating apoptosis-related proteins. Cancer Chemother Pharmacol 72: 789-798, 2013

27. Chou TC: Drug combination studies and their synergy quantification using the Chou-Talalay method. Cancer Res 70: 440-446, 2010.

28. Zhou Y, Hu Y, Yang M, Jat P, Li K, Lombardo Y, Xiong D, Coombes RC, Raguz S and Yagüe E: The miR-106b 25 cluster promotes bypass of doxorubicin-induced senescence and increase in motility and invasion by targeting the E-cadherin transcriptional activator EP300. Cell Death Differ 21: 462-474, 2014.

29. Ponti D, Costa A, Zaffaroni N, Pratesi G, Petrangolini G Coradini D, Pilotti S, Pierotti MA and Daidone MG: Isolation and in vitro propagation of tumorigenic breast cancer cells with stem/progenitor cell properties. Cancer Res 65: 5506-5511, 2005.

30. Grimshaw MJ, Cooper L, Papazisis K, Coleman JA, Bohnenkamp HR, Chiapero-Stanke L, Taylor-Papadimitriou J and Burchell JM: Mammosphere culture of metastatic breast cancer cells enriches for tumorigenic breast cancer cells. Breast Cancer Res 10: R52, 2008.

31. Ginestier C, Hur MH, Charafe-Jauffret E, Monville F, Dutcher J, Brown M, Jacquemier J, Viens P, Kleer CG, Liu S, et al: ALDH1 is a marker of normal and malignant human mammary stem cells and a predictor of poor clinical outcome. Cell Stem Cell 1: 555-567, 2007

32. Calcagno AM, Salcido CD, Gillet JP, Wu CP, Fostel JM, Mumau MD, Gottesman MM, Varticovski L and Ambudkar SV: Prolonged drug selection of breast cancer cells and enrichment of cancer stem cell characteristics. J Natl Cancer Inst 102: $1637-1652,2010$

33. Zhang X, Zhang S, Liu Y, Liu J, Ma Y, Zhu Y and Zhang J: Effects of the combination of RAD001 and docetaxel on breast cancer stem cells. Eur J Cancer 48: 1581-1592, 2012.

34. Miller TW, Balko JM and Arteaga CL: Phosphatidylinositol 3-kinase and antiestrogen resistance in breast cancer. J Clin Oncol 29: 4452-4461, 2011

35. Ghebeh H, Al-Khaldi S, Olabi S, Al-Dhfyan A, Al-Mohanna F, Barnawi R, Tulbah A, Al-Tweigeri T, Ajarim D and Al-Alwan M: Fascin is involved in the chemotherapeutic resistance of breast cancer cells predominantly via the PI3K/Akt pathway. Br J Cancer 111: 1552-1561, 2014

36. Liu Y, Zhang X, Liu J, Hou G, Zhang S and Zhang J: Everolimus in combination with letrozole inhibit human breast cancer MCF-7/Aro stem cells via PI3K/mTOR pathway: An experimental study. Tumour Biol 35: 1275-1286, 2014.

37. Zhu Y, Zhang X, Liu Y, Zhang S, Liu J, Ma Y and Zhang J: Antitumor effect of the mTOR inhibitor everolimus in combination with trastuzumab on human breast cancer stem cells in vitro and in vivo. Tumour Biol 33: 1349-1362, 2012.

38. Passacantilli I, Capurso G, Archibugi L, Calabretta S Caldarola S, Loreni F, Delle Fave G and Sette C: Combined therapy with RAD001 e BEZ235 overcomes resistance of PET immortalized cell lines to mTOR inhibition. Oncotarget 5: 5381-5391, 2014

39. Bareschino MA, Schettino C, Rossi A, Maione P, Sacco PC, Zeppa R and Gridelli C: Treatment of advanced non small cell lung cancer. J Thorac Dis 3: 122-133, 2011.
40. Harris CA, Ward RL, Dobbins TA, Drew AK and Pearson S: The efficacy of HER2-targeted agents in metastatic breast cancer: A meta-analysis. Ann Oncol 22: 1308-1317, 2011.

41. Holohan C, Van Schaeybroeck S, Longley DB and Johnston PG: Cancer drug resistance: An evolving paradigm. Nat Rev Cancer 13: 714-726, 2013.

42. Zhou J, Wulfkuhle J, Zhang H, Gu P, Yang Y, Deng J, Margolick JB, Liotta LA, Petricoin E III and Zhang Y: Activation of the PTEN/mTOR/STAT3 pathway in breast cancer stem-like cells is required for viability and maintenance. Proc Natl Acad Sci USA 104: 16158-16163, 2007.

43. Gupta PB, Onder TT, Jiang G, Tao K, Kuperwasser C, Weinberg RA and Lander ES: Identification of selective inhibitors of cancer stem cells by high-throughput screening. Cell 138: 645-659, 2009

44. Airiau K, Mahon FX, Josselin M, Jeanneteau M and Belloc F: $\mathrm{PI} 3 \mathrm{~K} / \mathrm{mTOR}$ pathway inhibitors sensitize chronic myeloid leukemia stem cells to nilotinib and restore the response of progenitors to nilotinib in the presence of stem cell factor. Cell Death Dis 4: e827, 2013.

45. Wang XQ, Ongkeko WM, Chen L, Yang ZF, Lu P, Chen KK, Lopez JP, Poon RT and Fan ST: Octamer 4 (Oct4) mediates chemotherapeutic drug resistance in liver cancer cells through a potential Oct4-AKT-ATP-binding cassette G2 pathway. Hepatology 52: 528-539, 2010.

46. Peiró G, Ortiz-Martínez F, Gallardo A, Pérez-Balaguer A, Sánchez-Payá J, Ponce JJ, Tibau A, López-Vilaro L, Escuin D, Adrover $\mathrm{E}$, et al: $\mathrm{Src}$, a potential target for overcoming trastuzumab resistance in HER2-positive breast carcinoma. Br J Cancer 111: 689-695, 2014

47. Pandya K, Wyatt D, Gallagher B, Shah D, Baker A, Bloodworth J, Zlobin A, Pannuti A, Green A, Ellis IO, et al: PKC $\alpha$ attenuates Jagged-1-mediated Notch signaling in ErbB-2-positive breast cancer to reverse trastuzumab resistance. Clin Cancer Res 22: 175-186, 2016.

48. Berns K, Horlings HM, Hennessy BT, Madiredjo M, Hijmans EM, Beelen K, Linn SC, Gonzalez-Angulo AM, Stemke-Hale K, Hauptmann M, et al: A functional genetic approach identifies the PI3K pathway as a major determinant of trastuzumab resistance in breast cancer. Cancer Cell 12: 395-402, 2007.

49. Zhu Y, Tian T, Zou J, Wang Q, Li Z, Li Y, Liu X, Dong B, $\mathrm{Li}$, Gao J, et al: Dual PI3K/mTOR inhibitor BEZ235 exerts extensive antitumor activity in HER2-positive gastric cancer. BMC Cancer 15: 894, 2015.

50. Elster N, Cremona M, Morgan C, Toomey S, Carr A, O'Grady A, Hennessy BT and Eustace AJ: A preclinical evaluation of the PI3K alpha/delta dominant inhibitor BAY 80-6946 in HER2-positive breast cancer models with acquired resistance to the HER2-targeted therapies trastuzumab and lapatinib. Breast Cancer Res Treat 149: 373-383, 2015.

51. Saura C, Bendell J, Jerusalem G, Su S, Ru Q, De Buck S, Mills D, Ruquet S, Bosch A, Urruticoechea A, et al: Phase Ib study of Buparlisib plus Trastuzumab in patients with HER2-positive advanced or metastatic breast cancer that has progressed on Trastuzumab-based therapy. Clin Cancer Res 20: 1935-1945, 2014.

52. Lehmann BD, Bauer JA, Chen X, Sanders ME, Chakravarthy AB, Shyr Y and Pietenpol JA: Identification of human triple-negative breast cancer subtypes and preclinical models for selection of targeted therapies. J Clin Invest 121: 2750-2767, 2011.

53. Rodler E, Korde L and Gralow J: Current treatment options in triple negative breast cancer. Breast Dis 32: 99-122, 2010.

54. Ibrahim YH, García-García C, Serra V,He L, Torres-Lockhart K, Prat A, Anton P, Cozar P, Guzmán M, Grueso J, et al: PI3K inhibition impairs BRCA $1 / 2$ expression and sensitizes BRCA-proficient triple-negative breast cancer to PARP inhibition. Cancer Discov 2: 1036-1047, 2012

55. Yunokawa M, Koizumi F, Kitamura Y, Katanasaka Y, Okamoto N, Kodaira M, Yonemori K, Shimizu C, Ando M, Masutomi K, et al: Efficacy of everolimus, a novel mTOR inhibitor, against basal-like triple-negative breast cancer cells. Cancer Sci 103: 1665-1671, 2012.

56. Singh J, Novik Y, Stein S, Volm M, Meyers M, Smith J, Omene C, Speyer J, Schneider R, Jhaveri K, et al: Phase 2 trial of everolimus and carboplatin combination in patients with triple negative metastatic breast cancer. Breast Cancer Res 16: R32, 2014.

57. Sun SY, Rosenberg LM, Wang X, Zhou Z, Yue P, Fu H and Khuri FR: Activation of Akt and eIF4E survival pathways by rapamycin-mediated mammalian target of rapamycin inhibition. Cancer Res 65: 7052-7058, 2005. 